\title{
Skin of the Chest
}

National Cancer Institute

\section{Source}

National Cancer Institute. Skin of the Chest. NCI Thesaurus. Code C161379.

The integument that covers the chest. 